\title{
L'élaboration du lexique français médiéval - variation diatopique, sélection et changement lexicaux
}

\author{
Videsott, P ; Glessgen, Martin-Dietrich
}

\begin{abstract}
The article examines some basic questions about the diatopic variation, the lexical selection and lexical change in Old French. In particular, the role, status and quantitative dimension of lexical regionalisms and lexical change is discussed. The selection processes in vocabulary, which in the different regions and political contexts of the langue d'oillare carried out with varying speed and intensity, are exemplified by a lexical analysis of two texts, a donation-document written in a Lorrain monastery in 1234 and a marriage contract established in the French Royal Chancery in 1301. While nearly a third of the vocabulary of the Lorraine document is no longer part of today's standard French, the vocabulary ofthe record of the Royal Chancery is in the 13th century already very close to that what will become the vocabulary of the future (and today's) standard.
\end{abstract}

DOI: https://doi.org/10.1515/roja-2017-0003

Posted at the Zurich Open Repository and Archive, University of Zurich ZORA URL: https://doi.org/10.5167/uzh-167230

Journal Article

Published Version

Originally published at:

Videsott, P; Glessgen, Martin-Dietrich (2017). L'élaboration du lexique français médiéval - variation diatopique, sélection et changement lexicaux. Romanistisches Jahrbuch, 68(1):64-89.

DOI: https://doi.org/10.1515/roja-2017-0003 


\title{
L'élaboration du lexique français médiéval - variation diatopique, sélection et changement lexicaux
}

\begin{abstract}
The article examines some basic questions about the diatopic variation, the lexical selection and lexical change in Old French. In particular, the role, status and quantitative dimension of lexical regionalisms and lexical change is discussed. The selection processes in vocabulary, which in the different regions and political contexts of the langue d'oil are carried out with varying speed and intensity, are exemplified by a lexical analysis of two texts, a donation-document written in a Lorrain monastery in 1234 and a marriage contract established in the French Royal Chancery in 1301. While nearly a third of the vocabulary of the Lorraine document is no longer part of today's standard French, the vocabulary of the record of the Royal Chancery is in the $13^{\text {th }}$ century already very close to that what will become the vocabulary of the future (and today's) standard.
\end{abstract}

\section{Lexique et variation diatopique dans la recherche}

L'on sait que l'élaboration linguistique porte sur tous les domaines du langage. Elle implique des processus de neutralisation et d'homogénéisation dans les domaines grapho-phonétique et morphologique et des processus de complexification dans les domaines morpho-syntaxique, syntaxique et lexical. Tout ceci vaut pleinement pour l'élaboration que le français a connue à travers l'époque médiévale, culminant dans la formation d'une variété standard à travers le $17^{\mathrm{e}}$ siècle $^{1}$.

Or, si dans les domaines grapho-phonétique, morphologique et syntaxique le processus d'élaboration, puis de standardisation du français a été relativement bien étudié, les processus de sélection lexicale n'ont jamais fait l'objet d'études très systématiques. Il y a certes, une multitude de travaux dédiés à des questions lexicales les plus diverses, liées à des textes donnés ou à des ensembles onomasio-

\footnotetext{
1 Ce processus se place, un peu comme en espagnol, entre ca 1480 et ca 1630 ; cf. Vachon 2010 qui étudie l'époque entre 1530 et 1650 et qui montre que les changements étudiés entre le moyen français et le français classique arrivent déjà à mi-chemin vers 1530 - ce qui suppose qu'ils ont dû débuter bien plus tôt - et qu'ils sont achevés vers 1630 .
}

DOI 10.1515/roja-2017-0003 
logiques. Mais la nature des trajectoires qui ont mené le vocabulaire des dialectes primaires de la Gaule septentrionale des $8^{\mathrm{e}}$ et $9^{\mathrm{e}}$ siècles au français standard du $16^{\mathrm{e}}$ siècle n'est connue que très partiellement.

Il nous ${ }^{2}$ semble emblématique que toutes les grammaires historiques comportent des chapitres sur la phonétique et la morphologie ${ }^{3}$; quant à la syntaxe, si elle est moins présente dans les grammaires historiques traditionnelles, elle est souvent traitée dans des ouvrages à part ${ }^{4}$. En revanche, aucune 'grammaire historique' ni même aucun ouvrage général sur le français médiéval ou sur l'histoire de cette langue comporte un traitement élargi de la lexicologie qui dépasserait les informations de base sur la provenance de certains mots du français moderne provenant des sub-, super- et adstrats et - de manière plus succincte - sur la (re)latinisation du vocabulaire médiéval.

Une analyse comparée des différentes grammaires historiques du français montre en effet que quand il est question de la physionomie du lexique français, l'aspect de sa composition étymologique (et donc les apports des différentes strates) domine largement sinon exclusivement ${ }^{5}$. Plus rarement, les auteurs tentent aussi d'identifier - toujours en relation avec les différentes strates - les domaines sémantiques qui produisent davantage de néologismes à une certaine époque ${ }^{6}$. L’aspect de la régionalité lexicale, qui nous sert de point d'appui ici,

2 La présente étude a été conçue par les deux auteurs qui ont rédigé à quatre mains quatre des cinq chapitres (1, 2, 3 et 5); " nous » désigne alors notre binôme. Le chapitre 4.1 a été rédigé par Martin Glessgen, 4.2 par Paul Videsott, changeant ainsi le référent du « nous ».

3 Il s'agit là de la répartition rendue 'classique' entre autres par les deux volumes de la « Altfranzösische Grammatik » de H. Rheinfelder ( $\left.{ }^{5} 1976 a,{ }^{2} 1976 b\right)$, cf. aussi Zink (1989, $\left.{ }^{3} 1991\right)$, mais déjà Schwan/Behrens $\left({ }^{11} 1919\right.$ [31897] : §1-282 « I. Teil : Lautlehre », § 283-430 « II. Teil : Formenlehre »).

4 Notamment l'excellente GNAF de Buridant 2000 ainsi que les deux ouvrages classiques de Ménard ${ }^{4} 1994$ [21973] et de Martin/Wilmet 1980 ou encore, bien avant, Foulet 1919 [31928].

5 Normalement, chaque strate est illustrée par un certain nombre de mots particulièrement significatifs qu'il a fourni au français (moderne); l'on présente alors : les différents substrats, en premier lieu le gaulois; les particularités de la strate latine (p.ex. des mots latins qui ne sont conservés que dans la Galloromania); les superstrats ; les différents adstrats correspondant aux emprunts des langues voisines après l'an 1000 : l'occitan, l'allemand, le néerlandais (avec leur dialectes), plus tard de l'italien, à l'époque moderne de l'anglais etc. (cf. la synthèse dans les chapitres correspondants dans la Romanische Sprachgeschichte : art. 136 [latin et Galloromania, Bork 2005], 138 [grec, Schlösser 2005], 141 [langues slaves, Buchi 2005], 143 [arabe, Kiesler 2005], 148 [allemand, néerlandais, Roegiest 2005], 150 et 151 [anglais, Görlach 2005 et Bartens 2005], 155 [emprunts à d'autres langues romanes, Trotter 2005b]).

6 Cf. p.ex. Kesselring 1970, 201-238. Le traitement le plus systématique du lexique se trouve dans la Französische Sprachgeschichte de Wolf (1979) qui prévoit un chapitre, bref, mais dense sur le lexique pour chaque époque traitée du latin jusqu'au $20^{\mathrm{e}}$ siècle (« Latein » : Vulgärlatein 33-35, 
entre en jeu seulement - dans les meilleurs des cas - pour les siècles suivant la diffusion du français standard, et donc dans une logique de différenciation tertiaire ${ }^{7}$. Pour la période antérieure à la formation de ce standard, donc avant le $16^{\mathrm{e}}$ siècle, l'aspect de la variation diatopique du lexique reste limité à l'énumération de certains 'mots vedette', qui en raison de leur état phonétique ne peuvent pas faire partie du lexique 'central's.

Dans cette optique se place par exemple le décompte ancien et souvent cité9 de Pierre Guiraud de 1968, qui détermine pour les différents siècles du Moyen Âge ce que l'auteur considère être des « prêts dialectaux dans le français » (Guiraud 1968,113 ) ; il en identifie en tout 171 pour la période qui nous concerne (41 pour le $12^{\mathrm{e}}$ siècle, 32 pour le $13^{\mathrm{e}}, 44$ pour le $14^{\mathrm{e}}$ et 54 pour le $15^{\mathrm{e}}$ ). Abstraction faite de l'erreur méthodologique de ce décompte - l'opposition entre 'dialectes' et 'français' postule, de manière implicite, l'existence d'un standard déjà au $12^{\mathrm{e}}$ siècle le chiffre global de 171 'dialectalismes' médiévaux supposés montre très clairement combien la variation lexicale dans l'espace a pu être sous-estimée et sousexploitée ; en effet, cette étude se trouve à des années-lumière de la réalité historique telle qu'elle peut être désormais établie (cf. infra 3.2).

\section{Raisons de l'impasse sur le lexique}

La question de la marginalité du lexique dans l'historiographie linguistique du français est naturellement complexe, d'autant plus que l'évolution du lexique comporte des aspects très divers :

a. la sélection que l'écrit opère parmi un vocabulaire quotidien à l'oral ; cette sélection génère pour la désignation des références quotidiennes un stock commun à la langue parlée et écrite ;

b. l'enrichissement du stock lexical de base par des mots d'un savoir spécialisé (religion, littérature profane, droit, sciences médico-biologiques et

Substrate/Superstrate 35-49, Erbwörter 50-52; «Altfranzösisch » : 842-1200, Lexikon 71-73, Normannen 73 ; 13. Jahrhundert, Lexikon (Buchwörter, Lehnwörter, Neubildungen) 82s. ; « Mittelfranzösisch » : Lexikon (Buchwörter, Lehnwörter, Dialektalismen, Neubildungen) 88-93; « Das 16. Jahrhundert » : Lexikon (Buchwörter, Lehnwörter, Neubildungen) 101-105; etc.

7 Cf. p.ex. Greub/Chambon 2008; Glessgen 2008b, 2970-2972.

8 Parmi les exemples les plus souvent cités on trouve caillou, caboche, falaise, quai, vergue = norm./pic. ; fablieau, flaque = pic. (Kesselring 1970, 200) ; v. aussi Duval 2007, 125 (norm. carpentier vs charpenteier, gueline vs géline etc.) ; 148-150 (lexique régional, p.ex. norm. babord, bord, cable, carguer etc.); 155s. (« Les dialectes dans la littérature comique »).

9 Cf. déjà Kesselring 1970, 200. 
mathématico-astronomiques ainsi que les autres multiples domaines du savoir médiéval) ; ici, l'écrit s'éloigne de la langue commune parlée et reflète des connaissances partagées par des groupes plus ou moins restreints de locuteurs ;

c. les emprunts aux langues de contact (flamand/néerlandais, francique/alémanique, anglais, occitan, franco-provençal etc.) autant dans le vocabulaire quotidien que dans le vocabulaire spécialisé ;

d. les nombreux emprunts formels et sémantiques au latin qui interviennent notamment dans le domaine des vocabulaires spécialisés.

Même si l'on fait abstraction des transformations purement grapho-phonétiques des lexèmes et si l'on se concentre sur les radicaux et affixes ainsi que sur le sens lexical, chacune de ces évolutions demande une approche différente ${ }^{10}$. Notre présente étude se concentre sur le premier aspect, le vocabulaire non-spécialisé (= a. et, partiellement, c.), et s'intéresse donc à une sélection à partir des variétés parlées de la langue.

Or, si une réflexion globale sur l'évolution du lexique 'général' du français au Moyen Âge est si peu développée aujourd'hui, ce n'est sans doute pas à cause de la complexité de la question : les évolutions morpho-syntaxiques sont loin d'être simples à traiter. Nous y voyons plutôt des raisons externes, liées aux traditions de la recherche. En ce sens, trois faisceaux fondamentaux semblent converger ici ; nous les commenterons par la suite.

\subsection{Absence de vues synthétiques sur le lexique}

Du point de vue de la lexicologie, nous nous trouvons face à une situation paradoxale : le français médiéval dispose d'un très grand nombre d'excellentes études sur le vocabulaire des textes (surtout, mais pas seulement littéraires) et également sur certains domaines onomasiologiques définis. Cette recherche éclatée a ensuite conflué vers une lexicographie historique qui peut être considérée comme exemplaire. Mais, ce qui est paradoxal, c'est que l'intégralité des énergies de la recherche sur le lexique s'est concentrée pendant plus d'un siècle sur ces deux ensembles, philologique et lexicographique, sans procéder à des interprétations lexicologiques globales ou à la rédaction de manuels synthétiques fournissant des aperçus généraux.

10 Ajoutons qu'une prise en considération des aspects syntagmatiques et phraséologiques ainsi que de la valence verbale compliquerait notablement le travail. 
Ainsi, le vocabulaire médiéval est très bien documenté mais cette documentation ne fournit que des informations très indirectes sur le processus de sélection $\mathrm{du}$ lexique standard qui est toutefois une interrogation fondamentale. Même si par exemple la documentation du FEW montre avec beaucoup de précision qu'un type lexical donné disparaît à une certaine époque, presque jamais le FEW n’a été utilisé comme source pour identifier les types lexicaux médiévaux qui ont disparu ou encore ceux qui se sont imposés.

Le cas est particulièrement flagrant pour la régionalité lexicale. Le seul dictionnaire médiéval qui donne des informations explicites sur la régionalité est le DMF, et encore d'une manière extrêmement partielle (Renders 2016). Cela vaut dans une mesure encore plus restreinte pour le DEAF(pré) qui se concentre essentiellement sur le marquage de l'anglo-normand, du franco-italien et des xénismes en zone frontalière (Tittel 2016). Enfin dans Gdf, TL et même FEW, de telles informations sont pleinement implicites : l'utilisateur doit déduire à partir des exemples donnés et de la localisation des textes, auteurs et copies, si les exemples couvrent ou non l'ensemble de l'espace oïlique (Greub 2016).

Il est certain qu'une interprétation généralisatrice du lexique est bien plus difficile que dans les domaines plus systémiques du langage, avec un nombre restreint d'oppositions fonctionnelles. Mais cela ne veut pas dire que de telles interprétations seraient impossibles. En effet, il existe les deux synthèses déjà anciennes de Schmitt 1974 - travail remarquable qui repose pleinement sur le FEW et qui poursuit des interrogations interprétatives globales, même s'il est sujet à caution d'un point de vue méthodologique - et Stefenelli 1981 - travail également remarquable, même si de lecture ardue -, mais ils sont restés isolés dans la production scientifique ${ }^{11}$.

\subsection{Absence d'une scriptologie lexicale}

Dans le domaine de la variation géolinguistique, un problème complémentaire réside dans le fait que l'analyse lexicale n'a jamais été un domaine considéré comme faisant partie de la scriptologie. Pour s'en convaincre, il suffit de feuilleter les ouvrages 'classiques’ de Gossen 1967, Goebl 1970, Dees 1980 et 1987, ou, plus récemment, Völker 2003 et Grübl 2014 : ces travaux contiennent tous des analyses sur des phénomènes grapho-phonétiques, morphologiques, parfois

11 Cf. toutefois le répertoire lexicographique récent de Carles 2017 traitant le lexique galloroman en contexte latin, culminant dans une interprétation évolutive du vocabulaire des premiers siècles. 
aussi syntaxiques ${ }^{12}$, mais jamais ne sont analysés des traits qui relèveraient $\mathrm{du}$ lexique. Les deux Atlas de Dees, par exemple, ne contiennent aucune carte 'lexicale', contrairement, p.ex. aux différentes analyses dialectométriques de l'ALF par Goebl.

Cet état de fait est la conséquence de l'approche scriptologique elle-même, qui est, comme on le sait, pleinement variationniste et ciblée sur des paramètres permettant la quantification ${ }^{13}$. Par ailleurs, la scriptologie privilégie une approche diatopique et, seulement dans une moindre mesure, diachronique et diastratique. Le lexique en revanche - tout comme la syntaxe - semble plutôt varier en fonction des genres textuels, et ainsi de façon diaphasique. Pour avoir une force probante du point de vue scriptologique, son analyse devrait intégrer le paramètre diaphasique bien davantage de ce qu'on ne l'a fait jusqu'à présent.

\subsection{Absence de concepts interprétatifs}

Enfin, la recherche a probablement aussi été freinée par le facteur idéologique sous-jacent au mythe du 'francien'. C'est la vision selon laquelle le français standard serait une pure et simple émanation du dialecte parlé de l'Île-de-France, 'parler directeur' qui aurait forgé le diasystème français à travers le Moyen Âge ${ }^{14}$. Ce mythe, difficile à déraciner encore aujourd'hui, a déterminé pendant longtemps la recherche sur la langue d'oïl. Or, dans cette vision la question de sélection du lexique ne se posait pas véritablement, car le lexique du français pouvait être expliqué comme étant le reflet du lexique du parler de l'île-de-France (la question de savoir si et dans quelle mesure la variété orale supposée à la base de la langue standard aurait déjà opéré des sélections lexicales, n’a jamais été posée dans ce contexte) avec quelques ajouts 'dialectaux' et surtout des ajouts de type diaphasique dans le vocabulaire spécialisé.

S’il est vrai qu'en italien, en espagnol ou en anglais il est effectivement possible d'identifier une base diatopique bien délimitée pour la future langue stan-

12 Un sujet 'classique' à cet égard est le respect ou non de la déclinaison bicasuelle (cf. Dees 1980, 206-208 ; Völker 2003, 169-192 et Grübl 2014, 236-243).

13 Cela est particulièrement facile dans le cas de variables diasystématiques qui reflètent des oppositions fonctionnelles, ce qui vaut surtout pour la grapho-phonétique et le marquage morphologique (cf. Glessgen 2012, 6). Une approche semblable est néanmoins possible aussi pour quelques secteurs du lexique. L’analyse scriptologique de Videsott 2009, 48 a p.ex. relevé la présence des trois types lexicaux DIES ( $v S$ DIURNUS), FRATER ( $v S$ FRATELLUS) et SOROR ( $v S$ SORELLA) en Italie du nord, sans pourtant les analyser en détail.

14 Cf. l'excellente synthèse de la tradition d'études chez Grübl (2014, 45-107) ainsi que le nouveau cadre évolutif proposé dans Glessgen (2017). 
dard - même si les processus de sélection lexicale restent extrêmement complexes (cf. Gardner 2016; Glessgen 2016; Schweickard 2016) -, pour le français cette idée est moins porteuse. Ici la situation est intermédiaire entre le cas de l'anglais et celui de l'allemand dont la codification médiévale est pleinement pluricentrique. Selon nous, la variété standard du français qui se constitue à Paris à travers la deuxième moitié du $13^{\mathrm{e}}$ siècle résulte d'une combinaison entre la mise à l'écrit du dialecte parlé ici - comme en anglais - et un processus de ‘koinéisation' et de neutralisation intra-régionale opérées au niveau de l'écrit (cf. Grübl 2014, 190-195) comme en allemand (cf. Glessgen 2017). Dans cette optique, la question de la variation diatopique du lexique français médiéval et celle des processus de sa sélection deviennent fondamentales.

\section{La régionalité lexicale du français au Moyen Âge : état actuel de la recherche}

Le mérite d'avoir attiré systématiquement l'attention des médiévistes sur la 'régionalité' lexicale revient surtout à un seul chercheur, Gilles Roques. En son honneur, la Société de Linguistique Romane a organisé en septembre 2015 un colloque à Zurich dédié à la régionalité lexicale au Moyen Âge. Ce colloque et le volume thématique qui en découle (Glessgen/Trotter 2016) jettent une lumière radicalement nouvelle sur la question et permettent de se prononcer sur les trajectoires générales de la régionalité en français, ce qui nous a amenés à repenser plus généralement les modalités de la sélection lexicale au Moyen Âge.

\subsection{Définition}

Un 'régionalisme' est « un lexème dont la forme et/ou le sens se caractérise par une diffusion régionale identifiable à l'intérieur de l'espace de la langue en question. Par 'forme', il faut entendre le radical et/ou l'affixe, non pas des éléments purement grapho-phonétiques ou flexionnels ${ }^{15}$. Les régionalismes lexicaux sont donc des lexèmes utilisés exclusivement ou de manière préférentielle dans une seule ou dans plusieurs régions données (= régionalismes exclusifs vs régionalismes de fréquence) » (Glessgen 2016, 3). Cette définition, développée à partir de celle de Gilles Roques, plus proche de la réalité d’observation («mot documenté

$\mathbf{1 5}$ Nous constatons en passant que ce type de variation régionale grapho-phonétique ou morphologique reste aussi largement sous-exploité. 
dans une ou plusieurs régions du domaine d'oïl sans être à diffusion générale ») met l'accent sur le fait que la 'régionalité' lexicale est un concept synchronique ${ }^{16}$. Concrètement, un 'régionalisme' ne peut être identifié que de manière inductive, c'est à dire en établissant le plus précisément possible le territoire d'emploi de chaque mot susceptible de n'être pas à diffusion générale. Interviennent alors la documentation médiévale, notamment non-littéraire, et les témoignages des dialectes modernes (cf. Chauveau 2016). La présence ou l'absence d'un lexème dans la langue moderne ne sont pas probantes pour une éventuelle régionalité médiévale, étant donné le grand nombre de mots anciens à large diffusion qui ont disparu à travers les siècles - une faible diffusion dans l'espace au Moyen Âge ne favorise toutefois pas l'intégration d'un terme dans la variété standard moderne.

Il est utile par ailleurs de différencier la nature des 'régionalismes' en fonction des concepts désignés : comme aujourd'hui, l'on peut distinguer au Moyen Âge des mots désignant des realia, à leur tour liés à une région donnée (comme des monnaies, mesures, redevances, droits ou objets agricoles et commerciaux) et des dénominations régionales d'un concept à diffusion générale (comme les exemples de la "terre en friche" et du "territoire" appelés terre treisse et fin dans le document lorrain étudié infra 4.1). L’analyse des documents anciens montre des différences importantes par rapport à ces deux catégories : déjà au $13^{\mathrm{e}}$ siècle, les rares régionalismes dans la chancellerie royale relèvent essentiellement du premier type, alors que les actes 'provinciaux' comportent de manière équitable les deux types.

\subsection{Quantification}

L'un des constats les plus importants par rapport à la recherche précédente est le nombre considérable de régionalismes lexicaux. Les dictionnaires du français médiéval répertorient pour cette langue un ensemble d'environ 80000 lexèmes. Sur ce chiffre global, il faut supposer un nombre important de mots spécialisés et également de latinismes qui appartiennent plutôt à l'écrit et qui ne sont régionalisés que très exceptionnellement (cf. supra 2, les catégories b. et c.). Le vocabulaire lié plus immédiatement aux références quotidiennes et à l'oral (la catégorie a.) et donc propice à une diffusion régionale (cf. infra 3.3) correspond seulement à une partie de ce lexique global, peut-être un tiers, certainement moins de la moitié.

Or, pour évaluer le nombre de régionalismes parmi cet ensemble nous disposons d'une première quantification précise, même si partielle, grâce à l'‘inventaire

16 D'un point de vue diachronique, l'aire de diffusion d'un régionalisme peut rester constante ou se réduire jusqu'à sa disparition ou encore s'agrandir jusqu'à sa généralisation. 
Roques' (Glessgen/Roques/Trotter 2016) ${ }^{17}$, seul inventaire spécifique de régionalismes lexicaux qui existe actuellement. Ce répertoire comporte en tout 2800 lexèmes régionaux. D’après les évaluations que nous avons pu faire, ce chiffre pourra être porté à une dimension d'environ 10000 lexèmes en exploitant pleinement la matière. Il s'agirait en effet de réunir les multiples informations à ce sujet qui se trouvent actuellement dispersées dans des milliers de glossaires, d'éditions de textes, d'études lexicologiques ou de compte rendus. Il s'agirait également d'exploiter plus intensément les sources documentaires des $14^{\mathrm{e}}$ et $15^{\mathrm{e}}$ siècles qui comportent de très nombreuses données lexicales connues jusqu'ici seulement par les dialectes modernes ${ }^{18}$. Mais un tel travail n'est possible que dans le cadre de la lexicographie de référence qui se place désormais sous les auspices de la lexicographie électronique, et donc évolutive. En fait, un mot médiéval ne peut pas être considéré a priori comme 'général' ou comme 'régional'; cette distinction doit être établie pour chaque mot (non spécialisé) de manière individuelle. En d'autres termes : il est erroné de considérer un mot comme répandu dans tout le diasystème médiéval avant de n'en avoir la preuve ${ }^{19}$.

L'un des mérites de l'inventaire Roques', malgré son caractère partiel, est de montrer sans équivoque combien le chiffre de 171 lexèmes régionaux de Pierre Guiraud est faux; c'est d'autant plus flagrant parce que bon nombre des formes retenues par Guiraud sont marquées seulement par leur phonétique. Les régionalismes véritables lexicaux se mesurent en milliers et non en dizaines. Il est donc désormais certain que la régionalité lexicale a joué un rôle essentiel dans la physionomie de la langue médiévale et dans l'élaboration de son écrit.

\subsection{Trajectoires}

Sur la base de nos constats, il devient également possible d'établir quelques idées générales sur les trajectoires de la régionalité en vue de la sélection lexicale pour

17 Cet inventaire, constitué dans le cadre du colloque de Zurich, prend appui sur l'intégralité des lexèmes identifiés comme régionaux par Gilles Roques dans ses travaux à travers les quatre dernières décennies.

18 Chauveau 2016 en a livré la preuve pour la Bretagne romane médiévale.

19 Ajoutons qu'il serait également fondamental d'établir une synthèse des données graphophonétiques et morphologiques, dont les informations sont également éclatées dans des milliers d'éditions et donc inutilisables pour des synthèses scriptologiques ou plus généralement linguistiques (cf. Glessgen 2012, 14-16). Pour les conditions pratiques pour arriver à un tel instrument (p.ex. la constitution d'un canon suffisamment grand de critères scriptologiques à analyser et quantifier impérativement dans chaque édition d'un texte médiéval) cf. Videsott (sous presse). 
la variété standard du français. Voici quelques observations qui nous semblent essentielles :

a. La lexicographie de référence ne prend (presque) pas en considération la régionalité, ce qui fausse le regard et ce qui complique la recherche dans ce domaine.

b. Les formes régionales qui parviennent à l'écrit ont leur origine dans l'oral ; celui-ci correspond au Moyen Âge de manière presque définitoire à des dialectes. La régionalité à l'écrit reflète donc des choix de l'oral dialectalisé.

c. L'écrit favorise de manière intrinsèque et générale des formes orales à diffusion large. Cela a plusieurs conséquences :

- les mots 'généraux', ayant une diffusion très large dans le diasystème sont particulièrement présents (et fréquents) dans les textes ;

- les mots dialectaux à diffusion restreinte (en usage seulement dans quelques villes ou villages) sont, quant à eux, très rares à l'écrit ;

- restent les mots qui ont une diffusion de type régionale, englobant parfois deux ou trois régions linguistiques (lorrain et franc-comtois; picard et wallon ; normand, Centre et Sud-Ouest) ; la fréquence relative et absolue de ces régionalismes dans les textes n'est pas très élevée, ce qui réduit leur visibilité, mais les mots régionaux sont néanmoins omniprésents même à l'écrit et ils témoignent alors d'une relation étroite avec l'oral.

d. La régionalité lexicale existe depuis la première époque ( $8^{\mathrm{e}}-10^{\mathrm{e}}$ siècle) et elle s'intensifie à travers le Moyen Âge, autant dans sa visibilité à l'écrit que vraisemblablement - dans la réalité du diasystème oral. Dans un mouvement inverse, la présence des mots régionaux « se réduit avec l'intensification de la standardisation et [ils] sont pour l'essentiel exclus de la variété standard écrite qui s'imposera aux $16^{\mathrm{e}}$ et $17^{\mathrm{e}}$ siècles » (Glessgen 2016, 23). L'on observe donc aux $14^{\mathrm{e}}$ et $15^{\mathrm{e}}$ siècles la coprésence contradictoire de textes déjà fortement dérégionalisés et d'autres textes, par exemple de type documentaire, avec l'apparition de mots antérieurement absents de l'écrit (mais certainement en usage à l'oral). Cette apparition reste, bien entendu, ponctuelle, puisque la tendance macroscopique de la standardisation est contraire à la régionalité.

e. La régionalité lexicale est tributaire des genres textuels : « les régionalismes apparaissent dans tous les genres textuels ; mais étant donné les grandes différences de contenu entre les genres, les mêmes régionalismes n’apparaissent pas dans tous les genres textuels (le syntagme lorrain agricole terre treisse [cf. infra 4.1] n'apparaîtra pas dans un texte littéraire, religieux ou médical). La valeur diasystématique, stylistique ou fonctionnelle des régionalismes est également différente selon les genres » $(i b ., 6)$. 
Il va sans dire que la variation lexicale ne répond pas seulement au paramètre de l'espace, mais également à tous les autres paramètres du diasystème - les aspects diastratiques (vocabulaire savant/de prestige), diaphasiques (vocabulaire spécialisé) et diamésiques - et aux différentes variables du système lexical - la distribution onomasiologique des concepts et leur spécificité sémantique, le degré de polysémie des formes, le réseau sémantique, le rôle de la dérivation, de la composition et de la phraséologie. Mais nous sommes désormais convaincus que « [l]e rôle des régionalismes dans le processus d'élaboration lexicale est particulièrement significatif, surtout en prenant en considération aussi les différents paramètres du diasystème et les genres textuels. L'étude des régionalismes fournit ainsi, au moins dans le cas du français, un apport épistémologique essentiel à la compréhension des mécanismes de sélection et de diffusion lexicale » (ib.).

\section{4 Études de cas : la régionalité et la sélection lexicale dans deux actes du $13^{\mathrm{e}}$ siècle}

Nous essaierons par la suite d'exemplifier ces interrogations à l'aide de deux textes des Plus anciens documents linguistiques galloromans (DocLing), l'un émanant du chapitre d'une abbaye de campagne, l'abbaye de Salival en Lorraine, l'autre de la chancellerie royale capétienne ${ }^{20}$. Nous les étudierons ici dans leur position par rapport au choix lexical de la variété standard.

\subsection{Abbaye de Salival}

Le premier document est un acte d'acensement de 1234 établi entre la petite abbaye de Salival (située à une vingtaine de kilomètres au sud-est de Metz) et deux paysans libres. Il s'agit du plus ancien document vernaculaire conservé dans le département de la Meurthe-et-Moselle, après la grande charte-loi de 1215 de Metz. La charte de Salival nous a servi à plusieurs reprises d'illustration pour les finalités

20 L'édition des deux textes a été téléchargée à partir du site des DocLing (www.rose.uzh.ch/ docling (comme il est possible de le faire pour chacun des 2200 actes qui s'y trouvent). Nous renvoyons au même site pour toute question concernant la transcription, présentation et édition des deux documents. L'acte abbatial a aussi été intégré dans le site pédagogique Ad Fontes de l'Université de Zurich (cf. 〈www.adfontes.uzh.ch〉; Glessgen 2011); le document royal, quant à lui, est également reproduit dans l'édition imprimée Videsott 2015, 378 s. 
du projet des Plus anciens documents ${ }^{21}$. Mais nous n'avons encore jamais analysé la physionomie lexicale de cet acte ${ }^{22}$.

Voici le texte dans le type d'édition 'mixte' (encodage médiéval et moderne combinés) que nous favorisons :

\section{chMM002}

1234 ( 25 mars-31 décembre) ou 1235 ( $1^{\mathrm{er}}$ janvier-24 mars)

Type de document : charte - acensement de terres

Objet : [2] L'abbé et le chapitre de Salival acensent à Wirrion et Houillon [3] treize journaux de terre dans le territoire de Juvelize [4] contre un cens de treize deniers et deux hémines de céréales [5] à payer annuellement sous peine de saisie de la terre. [6-9] La distribution et la position des treize journaux sont décrites plus en détail.

Auteur : non annoncé

Disposant : abbaye de Salival

Sceau : non annoncé

Bénéficiaire : abbaye Notre-Dame de Salival

Autres acteurs : Wirrion et Houillon, paysans de Juvelize

Rédacteur : scriptorium de l'abbaye Notre-Dame de Salival

Support : parchemin jadis scellé sur simple queue; $58 \times 141$

Lieu de conservation : AD MM H 1244, fonds de l'abbaye de Salival

1 Conue chose soit à-toz 2 que li abes et li chapitles de Salinvas · at laissié à Wirion $\backslash 2$ et Huillon, les dous freres de Geverlise, les anfanz Bertran Bacheler, 3 -XIII. jor $\backslash 3$ nas de terre treisse $\cdot$ en la fin de Geverlise $\cdot$ et à lor oirs $\cdot 4$ parmi $\cdot$ XIII - deniers de cens · et $\backslash 4 \cdot \mathrm{II} \cdot$ himas de blef · l'un d'avoine $\cdot$ l'autre de froment $\cdot \mathbf{5}$ et s'il ne paievent à jor $\backslash 5$ nomei à la feste sent Remi · à Giverlise, en la maison de Salinvas · que l'on se tan $\backslash 6$ roit à la terre · et ce que sus averoit · 6 Si est ensi devisee · q'au Tramble en $\backslash 7$ at $\cdot$ IIII jornas $\cdot$ un par lui $\cdot$ et $\cdot$ III ensemble $\cdot 7$ et en la voie de Hignicort en at $\backslash 8 \mathrm{~V} \cdot$ jornas $\cdot \mathrm{II} \cdot \mathrm{d}$ 'une part et $\cdot \mathrm{III} \cdot \mathrm{d}$ 'autre $\cdot \mathbf{8}$ et en la voie de Marsal -II · jornas · 19 aprés la terre les Vowes · 9 et en la voie de Donnereis · as Genoivres · en at $\backslash \mathbf{1 0} \cdot \mathrm{II} \cdot$ jornas $\cdot \mathbf{1 0} \mathrm{Ci}$ at mis li abes et li covenz de Salinvas son sael $\cdot$ en tesmoig \11nage de verité • $\mathbf{1 1}$ l'an que li miliaires corroit par ·M· et CC· et XXXIIII anz

21 Cf. Glessgen 2005 pour la réflexion sur les critères éditoriaux et l'analyse de la ponctuation, Glessgen 2011 dans le contexte des différents aspects de l'informatisation de notre corpus (cf. la note précédente) ou encore Glessgen 2008a, 441s. pour l'identification du rédacteur (justement, l'abbaye) ; cf. aussi Trotter 2005a, 273, 294 (édition).

22 D'une part parce que nous avons entrepris seulement depuis 2015 la lemmatisation du corpus global des Plus anciens documents dans le cadre du DEAF (cf. infra N30); d'autre part parce que nous n'avons commencé à réfléchir de manière plus systématique sur la question de la sélection lexicale entre le Moyen Âge et l'époque moderne qu'à la suite du colloque de Zurich sur la régionalité lexicale. 
Au-delà de l'apparence grapho-phonétique ${ }^{23}$, le lexique de ce bref document comporte toute une série de lexèmes - formes ou sens - qui n'ont pas survécu dans la variété standard du français. Nous enregistrons :

- deux mots régionaux qui n’ont jamais fait partie du français général et qui sont absents du français moderne :

(terre) treisse [3] adj. “(terre) en friche” ; cf. FEW 17, 400a s.v. *threosk "brachland/friche” : le substantif est attesté en wall., champ., lorr. ; l'adj. qui en dérive par conversion seulement dans les dialectes modernes, précisément de la Lorraine

hima[l] [4] s.m. "mesure de capacité" ; variante du dérivé eminal (frcomt., bourg., lorr.) formé à son tour sur emine "mesure ancienne de capacité"; cf. FEW 4, 402b s.v. HEMINA "art hohlmass": alothr. imal ${ }^{24}$

- deux lexèmes hors usage aujourd'hui :

miliaire [11] s.m. "millésime" : 1240-1644 (FEW 6/2, 91a s.v. MILLE) [dans le contexte que le miliaire courait qui est, même dans les chartes, plutôt régional]

oir [3] s.m. "héritier" : Roland - $17^{\mathrm{e}}$ siècle (FEW 4, 412b s.v. HERES)

- $\quad$ sept sens disparus dont un également régional :

blé [4] s.m. "céréales" : dans le document encore avec le sens général et hypéronymique de “céréales", aujourd'hui plutôt dans le sens spécifique de "froment” (cf. TLFi s.v. ; FEW 15/1, 126b et 127a s.v. ${ }^{\star}$ blād est imprécis)

denier [4] s.m. "monnaie valant un douzième du sou" : est en usage dans ce sens seulement comme terme historique (cf. TLFi s.v. ; FEW 3, 39b s.v. DENARIUS ne traite pas les différents sens de la forme)

deviser/diviser [6] v.tr. "répartir" (cf. TLFi s.v. ; FEW 3, 110b s.v. *DIVISARE est de nouveau imprécis quant à la sémantique)

fin [3] s.f., dans le syntagme prép. en la fin (de) "territoire” : le cas est complexe ; au pluriel, le lexème est habituel au Moyen Âge (cf. FEW 3, 561b : 13e s. - 1636); au singulier, il reste rare (cf. $i b$. N10), mais il fait partie des dialectes lorrains (et franc-comtois) modernes (cf. ib. : Moselle fi "confin ; territoire") ; il s'agit donc sans doute d'un usage régional déjà au Moyen Âge (cf. infra)

23 Comme nous l'avons dit, la variance graphématique et grapho-phonétique régionale est sans pertinence pour notre interrogation de sélection lexicale ; dans le présent exemple elle concerne notamment les formes suivantes (déjà relevées pour l'essentiel par Trotter 2005a, 273) :

- ['a] en syllabe ouverte > ['ej] : nomei "nommé” [5]

- absence de la consonne de transition entre [n’r] / [m'l] : tanroit [5]

- $\quad$ amuïssement de la liquide devant [-s] final : himas pour *himals [4] et jornas pour jornals [3]

- conservation graphique de $\langle t\rangle$ final dans la troisième personne du singulier avoir : at [6].

24 La forme himas au pluriel montre - en dehors l'amuïssement par assimilation de la liquide (cf. la note précédente) - dans la graphie un $\langle\mathrm{h}$ - $\rangle$ étymologique qui souligne que pour les scribes le lien du dérivé avec le mot de base hemina était encore transparent. 
jorna [l] [3] s.m. "mesure de terre, correspondant au travail d'une journée" : survit également dans ce sens comme terme historique (cf. TLFi s.v., Étymol. et Hist.; imprécis FEW 3, 103a s.v. DIURNUM)

maison [5] s.f. "couvent, monastère, abbaye” : Chrétien - 1340 (FEW 6/1, 236b s.v. MANSIO)

voie [7] s.f. "chemin, route” (à l'époque dans le sens concret, aujourd'hui au sens plus figuré ; cf. TLFi s.v. ; imprécis FEW 14, 371a s.v. vIA)

- $\quad$ notons également quelques phraséologismes aujourd'hui inhabituels comme à jour nommé [5].

Ce constat correspond à nos attentes après notre expérience avec les textes des Plus anciens documents : (i) les actes contiennent facilement l'un ou l'autre régionalisme, (ii) toute une série de formes ou sens disparus de la variété standard moderne et (iii) leur vocabulaire se concentre normalement dans le domaine de la vie et du droit agricoles.

En ce qui concerne la régionalité, il faut être prudent et précis : la densité des régionalismes lexicaux est très faible au niveau d'une charte individuelle. Un document de longueur moyenne comporte normalement un ou deux régionalismes, pas davantage. Il faut également noter que de tels régionalismes font souvent apparition dans un seul document de notre corpus :

- le dérivé imal s.m. (ici sous la forme himas pl.) est parfaitement isolé parmi les 2200 actes à côté des types simples amine (var. amene) surtout en Franche-Comté (40 occ.) et minne en Champagne ( 4 occ., plus mine [1 occ.] utilisé par la chancellerie royale);

- c'est également le cas de l'adjectif treisse - seule occurrence dans notre corpus - qui trouve toutefois une confirmation par deux occurrences du substantif qui en est à la base : lorr. tresques (1252, AbbSalival : en chans, en bois et en près des Bioncort, en tresques au bois de Gellacort) et trexes (1261, AbbSMBois [20 km au nord de Nancy, à l'ouest de Pont-à-Mousson] : toutes les terres, tant en trexes com en terres araules);

- dans ce sens, l'usage de fin au singulier est inhabituellement fréquent dans nos documents : la base de données dans sa deuxième version (2015) comporte non moins de 39 occurrences du lexème, surtout dans le syntagme en la fin de (ponctuellement dedens la fin, aprés la fin etc.) pour les corpus de la Marne (8×), de la Meuse (11×), de la Meurthe-et-Moselle (5x dont la charte de Salival), de la Haute-Marne (3x), des Vosges (2×), de la Haute-Saône ( $8 \times$ ) et, ponctuellement, du Jura (1×); cet usage est en revanche parfaitement absent des corpus de Douai et de la chancellerie royale (à l'exception d'une charte régionalement marquée en provenance de la Picardie, R 129703 3201 : de le fin dudit esquevinage), alors que l'usage pluriel paraît à plusieurs reprises dans un autre acte royal (R 1297061207 et sa copie RP 12970612 08), deux fois en Bourgogne (chNièvre) et seulement deux fois en Champagne et en Lorraine (chVosges 38 et chMarne 43).

Nous l'avons dit : la fréquence absolue et relative des régionalismes est pour la plupart faible dans les textes médiévaux qui favorisent très clairement des formes supra-régionales. Ce n'est donc qu'en réunissant des corpus importants et en les 
étudiant de près qu'il est possible d'avoir une idée de ce segment du vocabulaire : de manière très approximative, nous estimons actuellement que les 2200 chartes des Documents linguistiques dans la version de 2015 comportent un bon millier de régionalismes lexicaux ${ }^{25}$. Une seule charte n'est donc pas significative pour connaître ce segment du vocabulaire, mais l'addition de plusieurs centaines d'entre elles fournit une documentation consistante.

La faible fréquence des formes régionales à l'écrit ne doit pas induire en erreur : il s'agit de toute évidence de formes avec une présence constante à l'oral. Suivre la voie de ces régionalismes à travers leurs occurrences ponctuelles nous fournit ainsi une bien meilleure idée de la diversité de la langue parlée médiévale que nous concentrer sur les formes de l'écrit les plus fréquentes. Comme toujours en linguistique diachronique, en histoire ou en archéologie, ce sont les indices périphériques qui livrent une idée de la réalité au-delà de l'écrit, jamais les éléments qui sont placés au premier plan par les hommes de l'époque. Tout comme les graffiti de Pompéi, les mots régionaux dans les actes font ainsi apparaître une réalité linguistique inaccessible autrement.

$\mathrm{Au}$-delà de la question de la régionalité, le vocabulaire de notre charte reflète le jeu entre stabilité et transformation qui caractérise les trajectoires du lexique français depuis le Moyen Âge. Si sur les 42 mots lexicaux du texte, onze ne font pas partie de la langue standard actuelle, les autres trois quarts (31) témoignent d'une stabilité dans le vocabulaire à travers les presque huit siècles qui nous séparent de ce texte. Les lexèmes suivants sont en effet usuels encore aujourd'hui :

enfant, frère, jour, an, chose

abbé, couvent, chapitre, fête, saint

sceau, témoignage, vérité

terre, cens, part, avoine, froment

avoir, être, courir, laisser, mettre, nommer, payer, tenir

autre, connu, deux, ensemble, tous

Ces mots correspondent surtout à un vocabulaire des références quotidiennes où la variance est relativement faible, même si l'on remonte à l'Antiquité tardive ${ }^{26}$. Plus ponctuellement, ils reflètent une sélection également relativement ancienne

25 La version de 2016 avec, en plus, les chartes de la Côte d'Or et des cantons du Jura bernois et de Neuchâtel (pour l'essentiel oïliques) est plus riche en régionalismes.

26 Cf. INFANS, FRATER, ANNUS / ABBAS, CAPITULUM, SANCTUS / SIGILLUM [ ${ }^{\star}$ SIGELLUM], VERITAS / TERRA, CENSUS, PARS, AVENA, FRUMENTUM / HABERE, CURRERE, LAXARE, MITTERE, NOMINARE, TENERE ; avec un (léger) changement sémantique : CAUSA, CONVENTUS, PACARE ; avec une conversion : FESTUS adj. [DIES FESTA]. 
qui reste présente dans la variété standard moderne ${ }^{27}$. Un texte plus long et plus argumentatif comporterait aussi des formations avec une autre structure chronologique.

La stabilité d'un certain stock lexical héréditaire et non dérivé à travers deux millénaires est un des facteurs constitutifs des grandes trajectoires de l'évolution lexicale des langues romanes en général. Le nombre de ces mots est relativement restreint, mais il s'agit de concepts centraux d'un point de vue identitaire et cognitif (cf. Glessgen 2008b, 2951a). Leur concentration dans l'acensement lorrain de 1234 s'explique par la brièveté de l'acte en question et la simplicité de ses contenus qui se reflète immédiatement dans un vocabulaire et une syntaxe basiques.

Dans des actes plus longs du domaine oïlique oriental, la complexité autant du vocabulaire que celle de la syntaxe augmentent, ce qui réduit en même temps la part du lexique traditionnel invariant. Un seul exemple : un acte de vente de Pierrenot de Pierrecourt et de son fils Viot Huguenin au commandeur de l'église Saint-Antoine d'Aumônières à Nicolas Huin, scellé par la cour de Jean de Vergy, sénéchal de Bourgogne, et daté de l'extrême fin du $13^{\mathrm{e}}$ siècle (1298 n.st.) - l'acte 129 du corpus de la Haute-Saône des Plus anciens documents - comporte 132 mots lexicaux dont 27 ont un sens aujourd'hui inhabituel et 15 ont disparu ; près d'un tiers des lexèmes (42 de 132) se trouve donc en décalage avec le vocabulaire du français moderne ${ }^{28}$.

Les conclusions obtenues à partir de ces exemples lorrain et franc-comtois peuvent être élargies à l'ensemble des corpus 'départementaux' des Plus anciens documents : le vocabulaire français médiéval comporte de nombreux éléments qui sont encore en usage aujourd'hui, mais il comporte également un pourcentage important de mots inconnus voire incompréhensibles pour un locuteur du français actuel.

\subsection{Chancellerie royale}

Ayant passé plusieurs années à éditer et étudier les actes de la chancellerie royale, nous nous sommes demandé si cette conclusion était également valable pour ces documents ou si la vocation et l'importance supra-locales de cette chancellerie ex-

27 DIURNUS, TESTIMONIUM, *ESSERE/STARE.

28 Maria Selig nous a fait remarquer que la disparition de certains lexèmes pourrait être liée au fait qu'ils verbalisaient éventuellement des concepts liés à un contexte historique spécifique ; si cet argument est en effet valable pour les dénominations de mesures (émine, journal) ou de monnaie (denier) dans le document lorrain présenté, il ne concerne pas la plupart des concepts en question : chemin, territoire, répartir, terre en friche, céréales, héritier, couvent. 
cluait la présence de régionalismes voire même de lexèmes destinés à une courte longévité. Pour analyser ces questions, nous avons étudié de près un traité de mariage conclu par le roi Philippe le Bel et le duc de Bourgogne Robert pour leurs enfants respectifs. L'acte est daté du 28 mars 1301 et il s'agit du tout dernier acte de notre corpus de chartes royales vernaculaires $d u 13^{\mathrm{e}}$ siècle conservées en original (cf. Videsott 2015, 50).

\section{R 1301032801}

Château de Vincennes (Val-de-Marne) - 1301 (n.st.), 28 mars

Type de document : lettre patente en forme de charte

Objet : [1] Philippe [IV, dit le Bel], roi de France, [3] ayant obtenu la dispense ecclésiastique, [7] s'engage et promet [4] de donner en mariage à Marguerite, fille de Robert [II], duc de Bourgogne, son fils aîné Louis, quand il aura atteint l'âge de 14 ans, [5] ou à défaut, son second fils, Philippe. [10] Dans ce cas, le roi s'engage à obtenir la dispense nécessaire de l'Eglise, déjà obtenue pour le mariage prévu de Louis. [16] La reine Jeanne confirme le traité, [19] qui est scellé par les deux souverains, roi et [21] reine.

Auteur : Philippe [IV], roi de France

Disposant : Philippe [IV], roi de France

Sceau : Philippe [IV], roi de France ; Jeanne, reine de France et de Navarre, sa femme Bénéficiaire : Robert [II], duc de Bourgogne

Rédacteur : chancellerie royale

Support : original parchemin, $32,1 \times 21,1 \mathrm{~cm}$, scellé des sceaux de cire verte sur lacs de soie rouge et verte de Philippe [IV], roi de France et de Jeanne, reine de France et de Navarre, sa femme

Lieu de conservation : AD Côte-d’Or : B 290 nº 156 (A1, liasse $1^{\text {ère }}, \mathrm{n}^{\circ} 12$ )

Édition antérieure : Plancher II 1741, C-CI preuve 152

Verso, sur le repli à droite : Facta est collatio cum nota per me Bituris

1 Phelippes par la grace de Dyeu roys de France.'

2 Faisons savoir à touz, $\mathbf{3}$ que, comme dispensations soit otroiee et faite par l'Eglise de Rome ' 4 de $\backslash 2$ mariage faire ' entre nostre ainné fil ' Loys ' et Marguerite fille de nostre amé et foyal 'Robert duc de Borgoygne, ' 5 ou de Phelippe , nostre segont_né $\mid 3 \mathrm{fil}$, 'se il avenoit que li diz Loys morust avant que li diz mariages fust faiz de luy et de la dite Marguerite, ' $\mathbf{6}$ si comme veü et seü clerement havons ' 14 par lettres bullees de Rome, ' 7 nous prometons au dit duc pour luy et pour sa dite fille ' que, si tost comme li diz Loys havra passé quatorze anz, ' nous I 5 ferons et procurerons à nostre loyal povair ' que il prendra à femme et esposera en face de sainte Yglise la dite Marguerite.

8 Et se il avenoit, ' que ja ne soit, ' $\mid 6$ que li diz Loys morust avant que il heüst esposee la dite Marguerite en la maniere dessus dite, 9 nous prometons au dit duc, ausi comme devant, ' que nous ferons $\backslash 7$ et procurerons à nostre loyal povair que li diz Phelippes,' si tost comme il havra passé quatorze anz, prendra à femme 
et esposera en face de sainte Yglise, la dite 18 Marguerite, 10 en tele maniere que se en cestui mariage estoit ou pooit estre empeeschemenz de fillolage ou de cognation esperituel, ou de chose qui apartenist $\backslash 9$ ou poist apartenir à l'Yglise de Rome, ' 11 nous, aveuc le dit duc, ferons et procurerons à nostre loyal povair que dispensations en soit faite par l'Yglise de \10 Rome, 'à la fin que li diz mariages soit faiz entre le dit Phelippe et la dite Marguerite au plus tost qu'il porra estre faiz bonement.

12 Les choses dessus dites et chas \11cune par soi, ' nous prometons en bone foy, aut [sic!] dit duc por luy et pour sa dite fille, fermement tenir et garder, ' 13 et ne vendrons encontre, ne consen $\backslash 12$ tirons à venir, ' ainz tout empeeschement en bone foy en osterons. ' 14 Et donons pooir,' auctorité et commandement à nostre trés-chere compaingne Jehanne \13 par la grace de Dyeu royne de France, 15 qu'ele se puisse consentir et consente à toutes les choses dessus dites et à chascune d'iceles' et les promettoit $\backslash \mathbf{1 4}$ au dit duc, por luy et por sa dite fille, tenir et garder fermement en la maniere que nous li havons promis.

16 Et nous Jehanne par la grace $\backslash 15$ de Dyeu royne de France et de Navarre, ' dou pooir, ' de l'auctorité et dou commandement nostre trés-chier seigneur, Phelippe par la grace de Dyeu roy de \16 France à nous doné sus ce, ' 17 nous consentons à toutes les choses desus dites et à chascune par soy, ' et les prometons en bone foy fermement $\backslash \mathbf{1 7}$ tenir et garder, ' 18 et ne vendrons encontre,' ne consentirons à venir, ' ainz tout empeeschement en bone foy en osterons. '

19 En tesmoing de la quele $\backslash 18$ chose, ' nous Phelippes ' par la grace Dyeu roys de France dessus diz, ' havons fait mettre nostre seel en ces presentes lettres ' 20 et havons volu et consenti $\backslash 19$ que la dite royne, nostre trés-chiere compaigne face mettre son seel aveuc le nostre en iceles. '

21 Et nous Jehanne par la grace de Dyeu royne \20 dessus dite, ' par la volunté et par le consentement de nostre dit trés-chier seigneur le roy, ' havons fait mettre nostre seel avec le sien en ces lettres \21 22 qui furent faites et donees' au Boys de Vicennes, ' 23 le mardi aprés la annunciation nostre Seigneur, ' 24 l'an de grace , mil et trois cenz.'

L'analyse lexicale de cette charte amène à deux conclusions importantes : premièrement, au vu de toute la documentation disponible d'aujourd'hui, cet acte royal ne contient aucun régionalisme lexical. Or ce constat n'est pas - et de loin pas - un résultat isolé. Il est confirmé par l'analyse de l'intégralité du corpus 'royal'29 pour la question de la régionalité (cf. Videsott 2016). Sur les 140 documents analysés - tous plutôt longs et riches en vocabulaire -, seuls 14 contiennent

29 Nous nous référons à la totalité des 140 documents vernaculaires que nous avons identifiés comme émanant de la chancellerie royale et rédigés au $13^{\mathrm{e}}$ siècle. Il s'agit de 120 originaux et de 
des régionalismes. Les 16 lexèmes différents identifiés ${ }^{30}$ désignent pour l'essentiel des realia régionales : des mesures ou droits particuliers etc. Il semble donc possible de conclure que la chancellerie royale respectait jusqu'à un certain point ce type d'exigences 'dénominatives' concrètes, liées aux realia. Le statut de ces lexèmes serait donc celui d'emprunts' ou de 'citations' variationnelles (cf. Videsott 2016, 404).

Deuxième conclusion : la coïncidence des formes et sens lexicaux de notre document avec le français standard contemporain, à sept siècles de distance, est impressionnante. La comparaison n'est cependant pas aisée en raison du genre textuel juridique concerné dont les choix lexicaux et syntagmatiques se détachent nettement du langage général. Il faut également prendre en considération un décalage notable parmi les syntagmes, collocations, phraséologismes, dans le choix des prépositions et dans la valence verbale : dans ce domaine particulièrement caractéristique de la norme d'usage (dans le sens de Coseriu), les changements depuis le Moyen Âge restent extrêmement nombreux, autant d'ailleurs en français que dans d'autres langues à tradition historique comme l'italien, l'espagnol, l'allemand ou l'anglais. Ce phénomène est bien plus marqué dans le document royal que dans l'acte de Salival, dont la complexité de contenu et de structure est incomparablement plus réduite.

Le lecteur d'aujourd'hui se heurtera donc à des combinaisons inhabituelles aujourd'hui, comme :

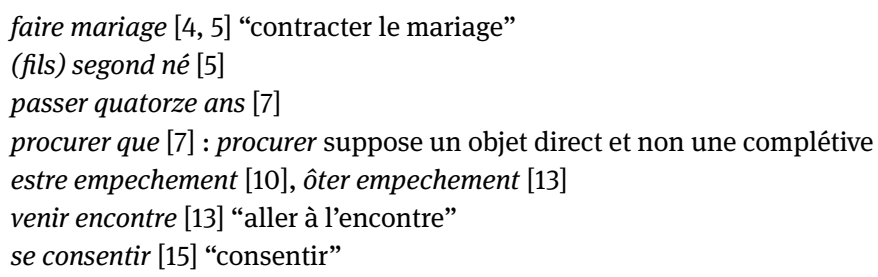

Notre interrogation porte toutefois ici de manière très ciblée sur la sélection des lexèmes individuels et non pas sur leurs combinaisons habituelles ou occasionnelles. Dans ce domaine, plus central ou systémique, la variance est moindre. En tout, nous avons relevé dans notre acte 116 lexèmes (correspondant à 183 formes différentes/'types' avant la lemmatisation et à 569 occurrences/'tokens') dont 82 mots lexicaux, 25 mots grammaticaux et 9 noms propres. Parmi les mots

20 copies d'actes royaux (en partie réalisées par d'autres institutions que la chancellerie royale). Pour plus de détails cf. Videsott 2015, 19-65.

30 S'ajoutent trois lexèmes - besenage, dechief, enaagé - que nous avons qualifiés pour l'instant de 'régionalismes potentiels' en raison de leur rareté. 
grammaticaux, nous constatons toujours un certain décalage par rapport à la langue actuelle :

ainz [13] prép. [après négation] “(mais) plutôt, (mais) au contraire” : afr. mfr. (FEW 24, 637a s.v. *ANTIUS ; cf. DMF)

sus [16] prép. "sur" : Eulalie - $16^{\mathrm{e}}$ siècle (FEW 12, 462b s.v. SŪRSUM)

cestui [10] dém. "ce" : afr. - 16e siècle (FEW 4, 820a, ǏsTE) ; icele [15, 20] "celle-ci” : St Léger Cotgr 1611 (FEW 4, 552a s.v. ĬLLE); li "lui” [15]

si tost comme [7] conj. "dès que" : afr. mfr. (FEW 13/2, 119b s.v. TosTUS)

au plus tost que [11] conj. "aussi tôt que” : afr. - 1636 (FEW 13/2, 120a s.v. TostUs)

en face de [7] loc. prép. “devant” (cf. TLFi s.v. D 2 : se marier en face de l'Église « il n'est guère usité dans cette phrase » (Ac 1932); imprécis FEW 3, 355b)

par soi [12] loc. prép. “individuellement” (cf. TLFi s.v. soi B 4d dans un autre usage ; Ø FEW)

Parmi les mots lexicaux en revanche, sur 82 lexèmes, seulement trois formes et deux sens ont disparu entre l'ancien français et le français moderne :

- formes disparues :

fillolage [10] s.m. "lien de parrain à filleul ; condition d'un parrain à l'égard de son filleul" : $12^{\mathrm{e}}-15^{\mathrm{e}}$ siècle (FEW 3, 520a s.v. FILIOLUS)

foyal [4] adj. "fidèle" : afr. mfr. (FEW 3, 502b, s.v. FIDELIS) ; remplacé par son doublet étymologique fidèle

ja [8] adv. "jamais” : afr. mfr. - Amyot [1513-1593] (FEW 5, 26b s.v. JAM)

- $\quad$ sens disparus :

apartenir (à) [10] v.intr. "relever (de)” : $13^{\mathrm{e}}$ siècle (FEW 25, 34a s.v. APPĚRTǏNĒRE)

bonement [11] adv. "correctement"

Ajoutons que certaines formes aujourd'hui sont hors usage, mais sont restées présentes au moins jusqu'au $19^{\mathrm{e}}$ siècle, comme notamment :

bullé [6] p.p. adjectivé : encore intégré dans le TLF, mais absent de PtRob 2010

dispensation [3, etc.] s.f. "dispense" : encore intégré dans le TLF, mais absent de PtRob 2010 (en) tesmoing (de) [19] s.m. “(en) témoignage (de)” : afr. - Ac 1798, 'vieux' dès Ac 1835 (FEW 13/1, 284b, TĚSTǏMŌNIUM)

Cela nous amène donc à seulement cinq sur ca 80 mots lexicaux de notre texte qui ont disparu à l'époque moderne ; si dans l'acte lorrain, relativement rudimentaire, un quart des lexèmes est aujourd'hui inconnu et dans une charte franc-comtoise, plus développée, un tiers, ici, il ne s'agit plus que d'un seizième ${ }^{31}$. La sélection

31 Notons que comme dans le document lorrain (cf. supra N29), la majorité des concepts en question ont gardé leur stabilité malgré la disparition des termes utilisés dans l'acte royal; seul fillolage et - parmi les mots aujourd'hui rares - bullé reflètent des concepts sans une verbalisa- 
lexicale de la chancellerie royale dans le document analysé est donc extrêmement proche de la norme actuelle.

Nous avons vérifié ce constat - si différent pour les chartes 'départementales' - par l'analyse analogue d'un certain nombre d'autres actes royaux, toujours dans l'idée d'établir leur décalage ou leur proximité par rapport au vocabulaire de la langue standard actuelle. Les effets restent identiques dans tous les cas que nous avons pu étudier, et ceci déjà pour le plus ancien document de la chancellerie royale, datant de 1241 (cf. Videsott 2010). Comme dans le document de 1301, cet acte est parfaitement exempt de régionalismes lexicaux ${ }^{32}$ et sur un ensemble de 72 mots lexicaux, il ne comporte que deux lexèmes qui diffèrent de la langue d'aujourd'hui.

Ce constat s'inscrit donc pleinement dans les résultats de l'analyse intégrale de notre corpus sous l'angle de sa régionalité lexicale : l'extrême rareté de mots régionaux - 16 sur les $c a 2000$ lexèmes de nos 140 actes $^{33}$ - est donc plus généralement corrélée avec l'absence relative de termes sans lendemain et spécifiques à la seule époque médiévale. À la lumière des données dont nous disposons actuellement, l'élaboration d'une variété exemplaire par la chancellerie royale au $13^{\mathrm{e}}$ siècle semble ainsi ne pas répondre à une logique chronologique, mais s'inscrire dans une stratégie propre à ce centre d'écriture et à la scripta en question.

\footnotetext{
tion courante dans la langue moderne. La ratio entre maintien et disparition est très semblable, malgré la différence évidente dans les thématiques traitées dans les deux actes.

32 L'acte comporte par contre un régionalisme morphologique avec la désinence -ains, -eins de la $1^{\mathrm{e}}$ pers. subj. prés. (Videsott 2016, 378). À propos de cette désinence, qui est considérée comme l'un des critères fondamentaux pour démontrer le caractère composite du standard français, cf. Grübl 2014, 125-137 (avec des renvois bibliographiques supplémentaires).

33 La lemmatisation du corpus des DocLing (réalisée en 2015 et 2016 par D. Kihaï, R. Bürli et M. Bergeron-Maguire, sous la direction de $M$. Glessgen) permet un décompte des lexèmes : sur le site des DocLing, il faut aller sur 'Les corpus textuels', puis cliquer sur 'Interrogations linguistiques [vers une autre interface]'; on arrive sur le site de la base de données Phoenix2 (en mode lecture), où il faut aller sur 'Assignment' [= la partie du logiciel qui permet des recherches sur les mots et graphèmes], puis sur 'Restrictions' et 'Corpus', cliquer sur 'Restrict' pour cocher ChRoy et ChRoyCp ; revenir sur 'Assign Occurrences' et faire une recherche (colonne à droite, case 'Query (REGEX)'), p.ex. sur les lemmes qui commencent avec $a$ - (recherche sur 'Lemmata', non pas sur 'Types' : ^a). Paraissent alors dans la colonne de droite tous les lemmes en question, ce qui permet de les compter.
} 


\section{Conclusions}

Si nous passons maintenant des cas concrets aux tendances plus générales, nous pouvons constater que, de toute évidence, la chancellerie royale opérait déjà au milieu et - dans de plus nombreux documents - à la fin du $13^{\mathrm{e}}$ siècle des choix lexicaux systématiques de type 'supra-régional' (ou 'inter-régional'). Les mots sélectionnés couvraient déjà assez bien la plus grande partie du domaine d'oïl continental. Il est par ailleurs probable, même si cela ne peut pas être prouvé, qu'il s'agissait de mots d'une certaine fréquence d'usage et qu'ils étaient intégrés dans différents genres textuels (ceci pouvant être néanmoins établi). En revanche, dans les autres lieux d'écriture du territoire d'oïl, le degré de 'standardisation' était bien moins avancé et ceux-ci faisaient apparaître une réelle diversité linguistique dans les usages.

Quant à la chancellerie royale, la qualité de diffusion large dans l'espace et sans doute aussi dans les contextes d'usage reflète donc une forme de 'compromis' à l'intérieur du diasystème. Si ce vocabulaire préfigure assez précisément la langue standard qui devait s'établir trois siècles plus tard, cela s'explique par deux raisons de nature radicalement différente : (i) d'abord, le fait que la chancellerie royale ait favorisé, justement, des lexèmes largement répandus auparavant rend probable déjà a priori la pérennité des mots en question ; (ii) par ailleurs, le rôle exemplaire qu'a joué cette variété dans le diasystème de l'écrit français a renforcé ultérieurement sa sélection lexicale. Il s'agit donc de la combinaison entre un fondement rationnel et une 'self fulfilling prophecy'34.

Or, pour les choix grapho-phonétiques et morphologiques, nous disposons d'éléments relativement précis concernant le processus de désintégration des scriptae oïliques régionales par l'adoption des variantes de la scripta parisienne et royale. Déjà Gossen (1957) avait commencé à établir un cadre de référence chronologique avec sa célèbre carte (p. 429) qui intégrait les données dont il disposait concernant l'abandon des scriptae régionales aux $14^{\mathrm{e}}$ et $15^{\mathrm{e}}$ siècles ; ce cadre nécessite bien entendu des précisions, même importantes, mais c'est une tâche dont la recherche est consciente et qu'elle poursuit actuellement ${ }^{35}$. Il nous semble évident par ailleurs que cette évolution correspond en dernier lieu à la

34 Cf. Glessgen 2017 pour une interprétation plus générale de l'élaboration d'une langue standard à travers les scriptae oïliques médiévales et pour la place de la Royauté dont l'importance a été mise en relief par Videsott (2010, 2015 etc.).

35 Les travaux déjà mentionnés de K. Grübl (p.ex. Grübl 2014, 342-351 et 2015, 28-30) sur un corpus de documents vernaculaires de Beauvais ont montré qu'il faut attendre la deuxième moitié $\mathrm{du} 14^{\mathrm{e}}$ siècle pour que la scripta parisienne ne se répercute sur la Picardie voisine. 
répercussion des chancelleries 'centrales' (chancellerie royale et Prévôté de Paris) sur le domaine d'oïl.

En revanche, pour le domaine du lexique, cette évolution n’a jamais été prise en considération. Par conséquent, une analyse systématique de l'écrit documentaire des $14^{\mathrm{e}}$ et $15^{\mathrm{e}}$ siècles sous cet aspect reste encore à mener. Une tâche à réaliser dans ce contexte serait notamment la comparaison des documents royaux avec des textes provenant de différentes régions - plus proches de l'îlede-France que la Lorraine ou la Franche-Comté - et avec des textes appartenant à différents genres textuels, littéraires, historiographiques et scientifiques. Il serait ainsi possible de vérifier sur une base plus large les modalités de l'uniformisation lexicale et la relation que celle-ci a entretenue avec la standardisation graphomorphologique.

En attendant, nos observations sur la régionalité lexicale et sur la différencialité du lexique médiéval et moderne fournissent dès à présent une vision d'ensemble plus cohérente et plus achevée des grandes évolutions du français depuis le Moyen Âge.

\section{Bibliographie}

Bartens, Angela (2005) : «Art. 151 : Contacts linguistiques : anglais et roman (hors d'Europe) », dans : RSG 2, 1708-1725.

Bork, Hans-Dieter (2005) : «Art. 136 : Sprachkontakte : Latein und Galloromania », dans : RSG 2, 1582-1590.

Buchi, Eva (2005) : «Art. 141 : Contacts linguistiques : langues slaves et langues romanes », dans : RSG 2, 1627-1639.

Buridant, Claude (2000) : Grammaire nouvelle de l'ancien français, Paris, Sedes.

Carles, Hélène (2017) : Trésor galloroman des origines (TGO). Les trajectoires étymologiques et géolinguistiques du lexique galloroman en contexte latin (ca 800 - 1120), Strasbourg, ÉLiPhi.

Chauveau, Jean-Paul (2016) : « Régionalismes médiévaux et dialectalismes contemporains en Haute-Bretagne », dans : Martin Glessgen/David Trotter (éds.), 131-166.

Dees, Anthonij (1980) : Atlas des formes et des constructions des chartes françaises du $13^{e}$ siècle, Tubingue, Niemeyer.

Dees, Anthonij (1987) : Atlas des formes linguistiques des textes littéraires de l'ancien français, Tubingue, Niemeyer.

DocLing ( $\left.{ }^{3} 2016\right)$ : Les plus anciens documents linguistiques galloromans (DocLing). Édition électronique, dirigée par Martin Glessgen, en partenariat avec Hélène Carles, Frédéric Duval et Paul Videsott. Troisième édition revue et fortement élargie, 〈www.rose.uzh.ch/ docling $\left({ }^{2} 2013,{ }^{1} 2009\right)$.

Duval, Frédéric (2007) : «Aux origines du français », dans : Alain Rey/Frédéric Duval/Gilles Siouffi, Mille ans de langue française : histoire d'une passion, Paris, Perrin, 9-454.

Foulet, Lucien $\left({ }^{3} 1928,{ }^{1} 1919\right)$ : Petite syntaxe de l'ancien français, Paris, Champion. 
Gardner, Anne-Christine (2016) : "The development of a lexical standard in English », dans : Martin Glessgen/David Trotter (éds.), 413-431.

Glessgen, Martin (2005) : «Editorische, lexikologische und graphematische Erschließung altfranzösischer Urkundentexte mit Hilfe von TUSTEP. Stand der Arbeiten », dans : Kurt Gärtner/Günter Holtus (éds.), Drittes Trierer Urkundensprachekolloquium (20.-22. Juni 2001), Trèves, THF, 91-107.

Glessgen, Martin (2008a) : "Les lieux d'écriture dans les chartes lorraines du XIII" siècle », dans : Revue de Linguistique Romane 72, 413-540.

Glessgen, Martin (2008b) : « Histoire interne du français (Europe) : lexique et formation des mots », dans : RSG 3, 2947-2974.

Glessgen, Martin (2011) : « Le long chemin d'une charte vers l'ère multimédiale », dans : Anja Overbeck/Wolfgang Schweickard/Harald Völker (éds.), Lexikon, Varietät, Philologie. Romanistische Studien. Günter Holtus zum 65. Geburtstag, Berlin, De Gruyter, 649-664.

Glessgen, Martin (2012) : «Trajectoires et perspectives en scriptologie romane », dans : Medioevo Romanzo 36, 5-23.

Glessgen, Martin (2016) : "La régionalité lexicale au Moyen Âge : hypothèses, constats et perspectives », dans : Martin Glessgen/David Trotter (éds.), 1-34.

Glessgen, Martin (2017) : « La genèse d'une norme en français au Moyen Âge : mythe et réalité du 'francien' ", dans : Revue de Linguistique Romane 81.

Glessgen, Martin/Roques, Gilles/Trotter, David (2016) : «Inventaire des régionalismes médiévaux français ", dans : Martin Glessgen/David Trotter (éds.), 473-635.

Glessgen, Martin/Trotter, David (éds.) (2016) : La régionalité lexicale du français au Moyen $\hat{A} g e$. Volume thématique issu du colloque de Zurich, organisé sous le haut patronage de la Société de Linguistique Romane (7/8 sept. 2015), Strasbourg, ÉLiPhi.

Goebl, Hans (1970) : Die normandische Urkundensprache. Ein Beitrag zur Kenntnis der nordfranzösischen Urkundensprachen des Mittelalters, Vienne, ÖAW.

Görlach, Manfred (2005) : «Art. 150 : Sprachkontakte : Englisch und Romanisch (Europa) », dans : RSG 2, 1699-1708.

Gossen, Carl Theodor (1957) : « Die Einheit der französischen Schriftsprache im 15. und 16. Jahrhundert », dans : Zeitschrift für romanische Philologie 73, 427-459.

Gossen, Carl Theodor (1967) : Französische Skriptastudien. Untersuchungen zu den nordfranzösischen Urkundensprachen des Mittelalters, Vienne, ÖAW.

Greub, Yan (2016) : «La régionalité dans la lexicographie du français médiéval (FEW, Gdf, TL) », dans : Martin Glessgen/David Trotter (éds.), 51-60.

Greub, Yan/Chambon, Jean-Pierre (2008) : « Histoire des variétés régionales dans la Romania : le français », dans : RSG 3, 2947-2974.

Grübl, Klaus (2014) : Varietätenkontakt und Standardisierung im mittelalterlichen Französisch. Theorie, Forschungsgeschichte und Untersuchung eines Urkundenkorpus aus Beauvais (1241-1455), Tubingue, Narr.

Grübl, Klaus (2015) : « Ce que les chartes nous apprennent sur la variation et le changement linguistique au Moyen Âge : l'exemple de la déclinaison bicasuelle de l'ancien français ", dans : Revue de Linguistique Romane 79, 5-38.

Guiraud, Pierre (1968) : Patois et dialectes français, Paris, PUF.

Kesselring, Wilhelm (1970): Die französische Sprache im Mittelalter, Tubingue, Narr.

Kesselring, Wilhelm (1981) : Dictionnaire chronologique du vocabulaire français. Le XVIe siècle, Heidelberg, Winter. 
Kesselring, Wilhelm (1989) : Dictionnaire chronologique de la langue française. Le XVII Siècle. T. 1. 1601-1606, Heidelberg, Winter.

Kiesler, Reinhard (2005) : «Art. 143 : Sprachkontakte : Arabisch und Galloromania », dans : RSG 2, 1648-1655.

Martin, Robert/Wilmet, Marc (1980) : Manuel du français du Moyen Âge. 1. Syntaxe du moyen français, Bordeaux, Sobodi.

Ménard, Philippe $\left({ }^{4} 1994,{ }^{2} 1973\right)$ : Manuel du français du Moyen Âge. 1. Syntaxe de l'ancien français, Bordeaux, Sobodi.

Renders, Pascale (2016) : « La régionalité lexicale du moyen français (1350-1500) dans le DMF », dans : Martin Glessgen/David Trotter (éds.), 85-96.

Rheinfelder, Hans ( $\left.{ }^{5} 1976 \mathrm{a}\right)$ : Altfranzösische Grammatik. 1. Teil : Lautlehre, Munich, Hueber.

Rheinfelder, Hans ( ${ }^{2} 1976$ b) : Altfranzösische Grammatik. 2. Teil : Morphologie, Munich, Hueber.

Roegiest, Eugeen (2005) : «Art. 148 : Contacts linguistiques : allemand/néerlandais et français », dans : RSG 2, 1685-1695.

RSG = Ernst, Gerhard/Glessgen, Martin-Dietrich/Schmitt, Christian/Schweickard, Wolfgang (éds.) (2003-2008) : Romanische Sprachgeschichte/Histoire linguistique de la Romania, 3 vols., Berlin/New York, De Gruyter.

Schlösser, Rainer (2005) : «Art. 138 : Sprachkontakte : Gelehrte Gräzismen in den romanischen Sprachen », dans : RSG 2, 1601-1610.

Schmitt, Christian (1974) : Die Sprachlandschaften der Galloromania. Eine lexikalische Studie zum Problem der Entstehung und Charakterisierung, Berne/Francfort-sur-le-Main, Lang.

Schwan, Eduard/Behrens, Dietrich $\left({ }^{11} 1919,{ }^{3} 1897\right)$ : Grammatik des Altfranzösischen. Laut- und Formenlehre, Leipzig, Riesland.

Schweickard, Wolfgang (2016) : " La régionalité lexicale en italien », dans : Martin Glessgen/David Trotter (éds.), 437-443.

Stefenelli, Arnulf (1981) : Geschichte des französischen Kernwortschatzes, Berlin, Schmidt.

Tittel, Sabine (2016) : " La régionalité lexicale de l'ancien français (ca 1100 - ca 1350) : une enquête sur la base du Dictionnaire étymologique de l'ancien français », dans : Martin Glessgen/David Trotter (éds.), 61-83.

Trotter, David (2005a) : « Diastratische und diaphasische Variation. Normierungstendenz und Unabhängigkeit in lothringischen Dokumenten des Mittelalters », dans : Kurt Gärtner/Günter Holtus (éds.), Drittes Trierer Urkundensprachekolloquium (20.-22. Juni 2001), Trèves, THF, 245-322.

Trotter, David (2005b) : «Art. 155 : Contacts linguistiques intraromans : roman et français, occi$\tan »$, dans : RSG 2, 1776-1785.

Vachon, Claire (2010) : Le changement linguistique au XVI siècle. Une étude basée sur des textes littéraires français. Préface de David Trotter, Strasbourg, SLR/ÉLiPhi.

Videsott, Paul (2009) : Padania scrittologica. Analisi scrittologiche e scrittometriche di testi in italiano settentrionale antico dalle origini al 1525. Con 15 figure, 60 tabelle e 159 cartine, Tubingue, Niemeyer.

Videsott, Paul (2010) : «À propos du plus ancien document en français de la chancellerie royale capétienne ", dans : Bibliothèque de l'École des Chartes 168, 61-81.

Videsott, Paul (2015) : Les plus anciens documents en français de la chancellerie royale capétienne (1241-1300). Présentation et édition, Strasbourg, ÉLiPhi.

Videsott, Paul (2016) : « La chancellerie royale et la régionalité lexicale », dans : Martin Glessgen/David Trotter (éds.), 377-410. 
Videsott, Paul (sous presse) : «La diatopie (la scripta) », dans : Frédéric Duval/Céline Guillot/ Fabio Zinelli (éds.), Les introductions linguistiques aux éditions de textes, Paris, Garnier. Völker, Harald (2003) : Skripta und Variation. Untersuchungen zur Negation und zur Substantivflexion in altfranzösischen Urkunden der Grafschaft Luxemburg (1237-1281), Tubingue, Niemeyer.

Wolf, Heinz Jürgen (1979) : Französische Sprachgeschichte, Heidelberg, Quelle \& Meyer. Zink, Gaston (1989) : Morphologie du français médiéval, Paris, PUF.

Zink, Gaston (31991) : Phonétique historique du français, Paris, PUF. 